\title{
Pressure Induced Structural Phase Transition, Metallization and Superconductivity in SodiumIodide (NaI)
}

\author{
Y. Ramola ${ }^{1}$, J. Merlinebetsy ${ }^{1}$, C. Nirmala Louis ${ }^{1, *}$, A. Amalraj ${ }^{2}$ \\ ${ }^{1}$ Research Department of Physics, Holy Cross College (Autonomous), Manonmaniam Sundaranar University, India \\ ${ }^{2}$ St. Jerome's College, Ananthanadarkudy, Manonmaniam Sundaranar University, India
}

Copyright $\bigcirc 2019$ by authors, all rights reserved. Authors agree that this article remains permanently open access under the terms of the Creative Commons Attribution License 4.0 International License

\begin{abstract}
The structural phase transition and metallization of the simple alkali iodide, sodium iodide $(\mathrm{NaI})$ is investigated through their band structures. The band structure, density of states (DOS) and total energy are computed as a function of volume for both $\mathrm{NaCl}$ and $\mathrm{CsCl}$ phases using the full potential linear muffin-tin orbital (FP-LMTO) method. The phase transition pressure $\left(P_{\mathrm{T}}\right)$ and the corresponding reduced volume $\left(V_{\mathrm{T}}\right)$ estimated in our calculation are $0.038 \mathrm{Mbar}$ and 0.89 respectively. The metallization pressure $\mathrm{P}_{\mathrm{M}}$ is $2.6259 \mathrm{Mbar}$ and the corresponding reduced volume $\left(V_{\mathrm{M}}\right)$ is 0.36 . The results of the metallization pressure in $\mathrm{NaI}$ are compared with that of other alkali iodide spotassium iodide (KI) and RbI. It is found, that the charge transfer from $s$ and $p$ states to the $d$ state will cause metallization and the metallization pressure increases with a decrease of the lattice constant. It is also confirmed that the metallization and structural phase transition do not occur simultaneously in ionic compounds.
\end{abstract}

Keywords Band Structure, Metallization, Structural Phase Transition, NaI, Density of States

\section{Introduction}

The physical properties of materials undergo a variety of changes when they are subjected to high pressure [1]. The increase of pressure means the significant decrease in volume, which results in the change of electronic states and crystal structure [2].The modern development in diamond anvil cell [3-11] enables the experimentalist to perform the investigation at very high value of pressure( 5 Mbar). Already twenty three new elemental superconductors were found at high pressure. In this list new compounds are now being included. One such compound is the simple ionic salt CsI [3]. This material is a testing ground both for photo converters [3].The metallization in CsI is experimentally achieved by Vohra [12]. But Eremets experimentally find the evidence for the metallization and superconductivity of CsI at high pressure. The ionic salt CsI is a metal under high pressure and as the pressure increases superconductivity sets in CsI [4]. In KI, Wier et al [13] experimentally reported the structural phase transition and metallization. In the present paper, we give more extensive discussion about the band structure, density of states and electronic charge distributions as a function of reduced volume. The structural phase transition, stability of the phase, equilibrium bulk modulus, metallization and superconductivity are reinvestigated and critically analyzed. The pressure dependence of the band gap and enthalpy calculations is performed at various high pressures. We give the details of the calculational procedure and electronic band structure corresponding to various pressure in section 2 . In Section 3, the ground-state properties, structural phase transition and metallization are discussed. Concluding remarks are given in Section 4. These results lead us to expect superconductivity in other alkali halides under high pressure, especially those alkali iodides which have already become metals [1].

\section{Band Structure and Density of States}

\subsection{Calculational Procedure}

Sodium iodide crystallizes in rock salt structure under ambient conditions and undergoes structural phase transition from $\mathrm{NaCl}$ to $\mathrm{CsCl}$ structure. The electronic band structure calculations were performed for $\mathrm{NaI}$ corresponding to different reduced volumes both in $\mathrm{NaCl}$ to $\mathrm{CsCl}$ structures, by the first-principle linear muffin-tin orbital (FP-LMTO) method. We have used FP-LMTO method with in generalized gradient approximation (GGA) $[14,15]$. It is based on the idea that one electron states of solids can be written as combinations of a small number of 
states of the constituent atoms. Also the crystal potential is approximated by a series of non-overlapping atomic like spherically symmetric potentials and a constant potential between the spheres. This method has the advantage that the theory and the result are very easy to understand. For this reason most results of the electronic structures computed are interpreted in the light of full potential theory [16]. This full potential model assumes the solution to the time-independent single electron Schrödinger equation $\Psi$ is well approximated by a linear combination of atomic orbitals. In this linear method, functions constructed from partial waves and their first energy derivatives obtained within the muffin-tin approximation are used as fixed basis. The chemical content of this technique acts as a guide to the non-specialist who wants to perform band structure calculations of his own. The problem of electronic structure involves the computation of eigen states for an infinite number of interacting electrons. This leads to the estimation called one electron approximation, which describes each electron as an impendent particle moving in the mean field of the order electrons plus the field of the nuclei[16].By standard variation technique one can obtain a set of linear equations, in terms of the Hamiltonian and overlap matrices to determine the eigen $\mathrm{E}$ and the expansion coefficients. Here the one electron energies are found by a single diagonalisation of the secular matrix [14,15].

We give here only the calculational details. The electronic configurations of $\mathrm{Na}$ is $[\mathrm{Ne}] 3 \mathrm{~s}^{1}(Z=11)$ and for it is $[\mathrm{Kr}] 4 \mathrm{~d}^{10} 5 \mathrm{~s}^{2} 5 \mathrm{p}^{5}(Z=53)$. The valence electronic configurations of $\mathrm{Na}$ and $\mathrm{I}$ are $3 \mathrm{~s}^{1}$ and $5 \mathrm{~s}^{2} 5 \mathrm{p}^{5}$ respectively. There are 8 electrons contribute to the valence band. The final energy convergence is within $10^{-5} \mathrm{Ry}$. The calculated total energies were fitted to Murnaghan's equation of state (EOS) [17], to determine the bulk modulus and pressure derivative of bulk modulus $\mathrm{B}_{0}{ }^{1}$. We have obtained,

(i) Normal pressure band structure and density of states $\mathrm{NaI}$ (with $\mathrm{NaCl}$ structure)

(ii) High pressure band structure and density of states of $\mathrm{NaI}$ (with $\mathrm{CsCl}$ structure)

The calculated total energies are fitted to Murnaghan's equation of states (EOS), to determine the pressure derivative of bulk modulus $\mathrm{B}_{0}{ }^{1}$, phase- transition pressure and other ground - state pressure and other ground state properties $[18,19]$. The bulk modulus is a property of the material which defines its resistance to volume change when compressed. The cause for phase transition and the relation phase transition pressure and atomic radii are deduced from band structure investigation [10].

\subsection{Band Structure and Density of States of NaI under Pressure}

\subsubsection{Band Structure and Density of States at Normal Pressure}

The normal pressure band structure of $\mathrm{NaI}$ (for $\mathrm{NaCl}$ structure) is given in Fig.1. The overall topology of the band structure at $\mathrm{V} / \mathrm{Vo}=1$ is same for previous calculation. The three bands appearing just below the Fermi energy $E_{F}$ are from Na-3s ${ }^{1}$ and I- $5 s^{2}, 5 p^{5}$ electrons of NaI (Fig. 1). The empty conduction bands above the Fermi level are due to $3 \mathrm{p}^{0}, 3 \mathrm{~d}^{0}$ states of $\mathrm{Na}$ and $5 \mathrm{~d}^{0}, 6 \mathrm{~s}^{0}, 6 \mathrm{p}^{0}$ states of I(Fig. 1). At normal condition, the band gap Eg is found to be $0.2480 \mathrm{Ry}$ $(3.373 \mathrm{eV})$ for NaI. The general features of band structures are similar to previous calculations. From our calculation, $\mathrm{NaI}$ is a direct band gap insulator at normal pressure. The density of histograms of $\mathrm{NaI}$ at normal pressure are given inFig. 2. From the histogram, it is seen that at normal pressure the short spikes near $\mathrm{E}_{\mathrm{F}}$ are due to $\mathrm{Na}-3 \mathrm{~s}^{1}, \mathrm{I}-5 \mathrm{p}^{5}$ electrons (Fig. 2). The short spikes above the $\mathrm{E}_{\mathrm{F}}$ are due to the $3 \mathrm{p}^{0}, 3 \mathrm{~d}^{0}$ and $5 \mathrm{~d}^{0}, 6 \mathrm{~s}^{0}, 6 \mathrm{p}^{0}$ states of NaI (Fig. 2). At normal density of states histogram, the band gap Eg is found to be $0.2480 \mathrm{Ry}(3.373 \mathrm{eV})$ for NaI. This value is agreement with the experimental value of $3.36 \mathrm{eV}$. The general features of density of states are similar to previous calculations [1].

\subsubsection{Band Structure and Density of States at High Pressure}

The high pressure band structure of $\mathrm{NaI}$ (for $\mathrm{CsCl}$ structure) is given in Fig. 3. Fermi level is indicated by dotted horizontal line. The overall topology of the band structure at $\mathrm{V} / \mathrm{Vo}=0.36$ is same as Fig. 1. But Fig. 3 represents metallic $\mathrm{NaI}$ and Fig. 1 represents insulating NaI. The triplet bands which are positioned at the bottom of valence band arise $3 \mathrm{~s}^{1}$ electrons of $\mathrm{Na}$ (Fig. 3). A single band nearer to the triplet bands is due to $\mathrm{I}-5 \mathrm{~s}^{2}$ electrons (Fig. 3). The three bands appearing just below the Fermi energy $E_{F}$ and touching Fermi energy $E_{F}$ are from Na-3s $s^{1}$ and I-5 $p^{5}$ electrons of NaI (Fig. 3). The filled conduction bands above the Fermi level are due to $3 \mathrm{p}^{0}, 3 \mathrm{~d}^{0}$ states of $\mathrm{Na}$ and $5 \mathrm{~d}^{0}, 6 \mathrm{~s}^{0}$, $6 \mathrm{p}^{0}$ states of I (Fig. 3). In NaI, metallization takes place by the indirect closure (Fig. 3) of band gap between valence band maxium of $I$ point and conduction band minimum at the $\mathrm{H}$ point. The metallization volume of $\mathrm{NaI}$ is $\mathrm{V} / \mathrm{Vo}=0.36$, which corresponds to the pressure $\mathrm{P}_{\mathrm{M}}=2.6259 \mathrm{Mbar}$. The general features of high pressure band structure is similar to previous calculations [8-12]. From our calculation, in $\mathrm{NaI}$ direct closure of band gap occurs.

The density of states histograms of $\mathrm{NaI}$ at high pressure is given in Fig. 4. At this pressure $\mathrm{NaI}$ is in $\mathrm{CsCl}$ structure. From the histogram, it is seen that at high pressure the levels arising from Na-3s ${ }^{1}$ (Fig. 4) electrons give a longest spike with fine width. The extended spike near the origin is due to $\mathrm{I}-5 \mathrm{p}^{5}$ electrons and the dumpy spikes near $\mathrm{E}_{\mathrm{F}}$ are due to Na-3s ${ }^{1}, I-5 p^{5}$ electrons (Fig. 4). The petite spikes above the $E_{F}$ are due to the $3 p^{0}, 3 d^{0}$ and $5 d^{0}, 6 s^{0}, 6 p^{0}$ states of $\mathrm{NaI}$ (Fig. 4). In Fig. 4 the heights of the spikes are considerably reduced when compared to Fig. 2. The reason is when pressure is increased $E_{F}$ increases whereas no density of states is available at the Fermi level up to metallization pressure. There are appreciable values for DOS at $\mathrm{V} / \mathrm{V}_{\mathrm{O}}=$ 0.36 (Fig. 4) indicating metallization in NaI. 


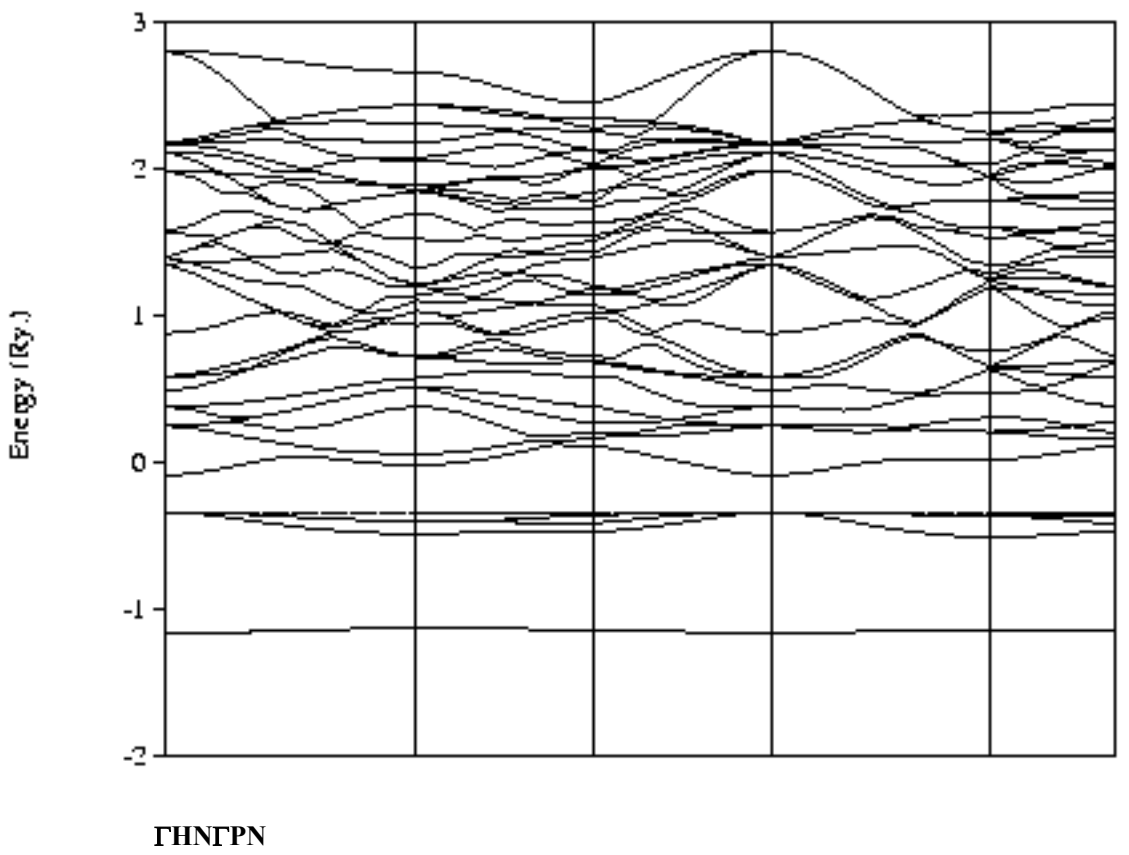

Figure 1. Band structure of $\mathrm{NaI}$ at $\mathrm{V} / \mathrm{Vo}=1.0(\mathrm{NaCl}$ structure $)$

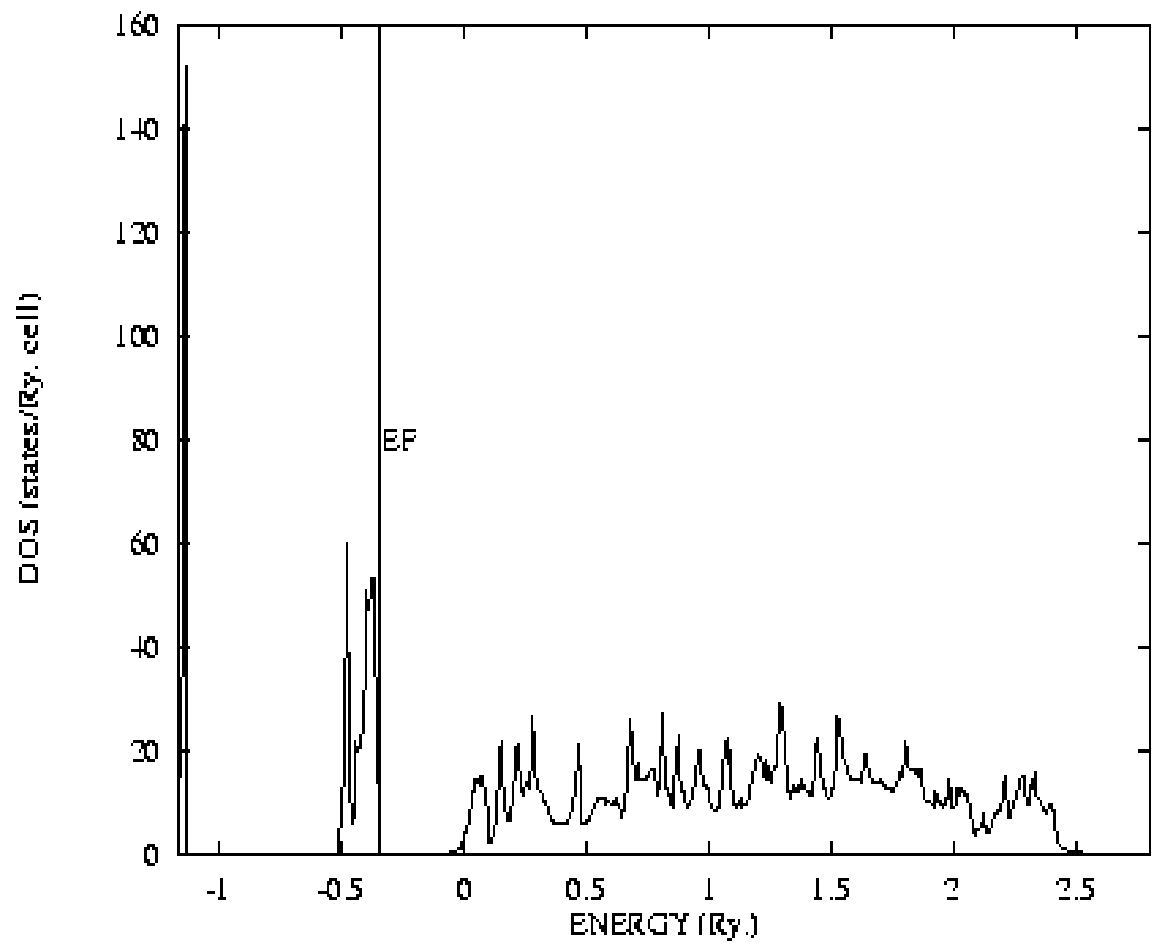

Figure 2. Density of states of $\mathrm{NaI}$ at $\mathrm{V} / \mathrm{Vo}=1.0(\mathrm{NaCl}$ structure $)$ 


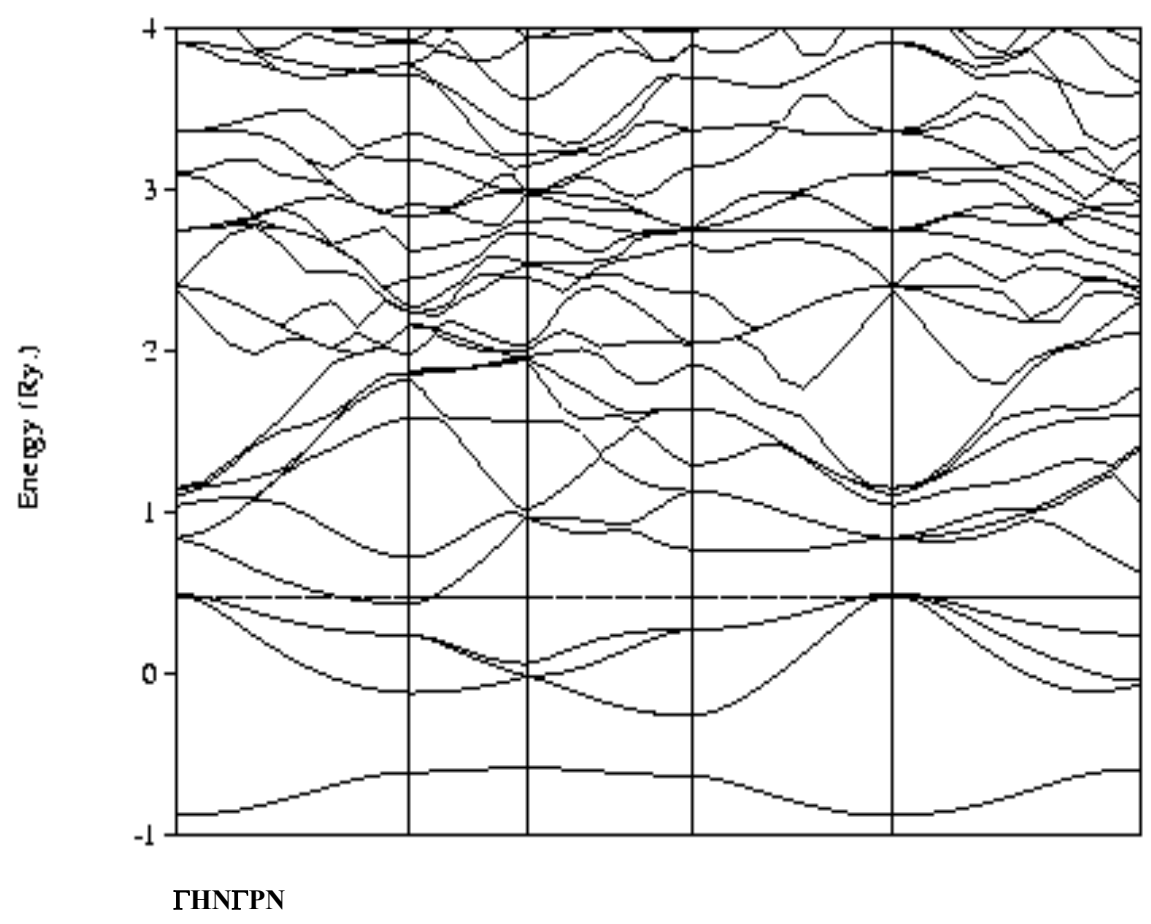

Figure 3. Band structure of $\mathrm{NaI}$ at $\mathrm{V} / \mathrm{Vo}=0.36(\mathrm{CsCl}$ structure $)$

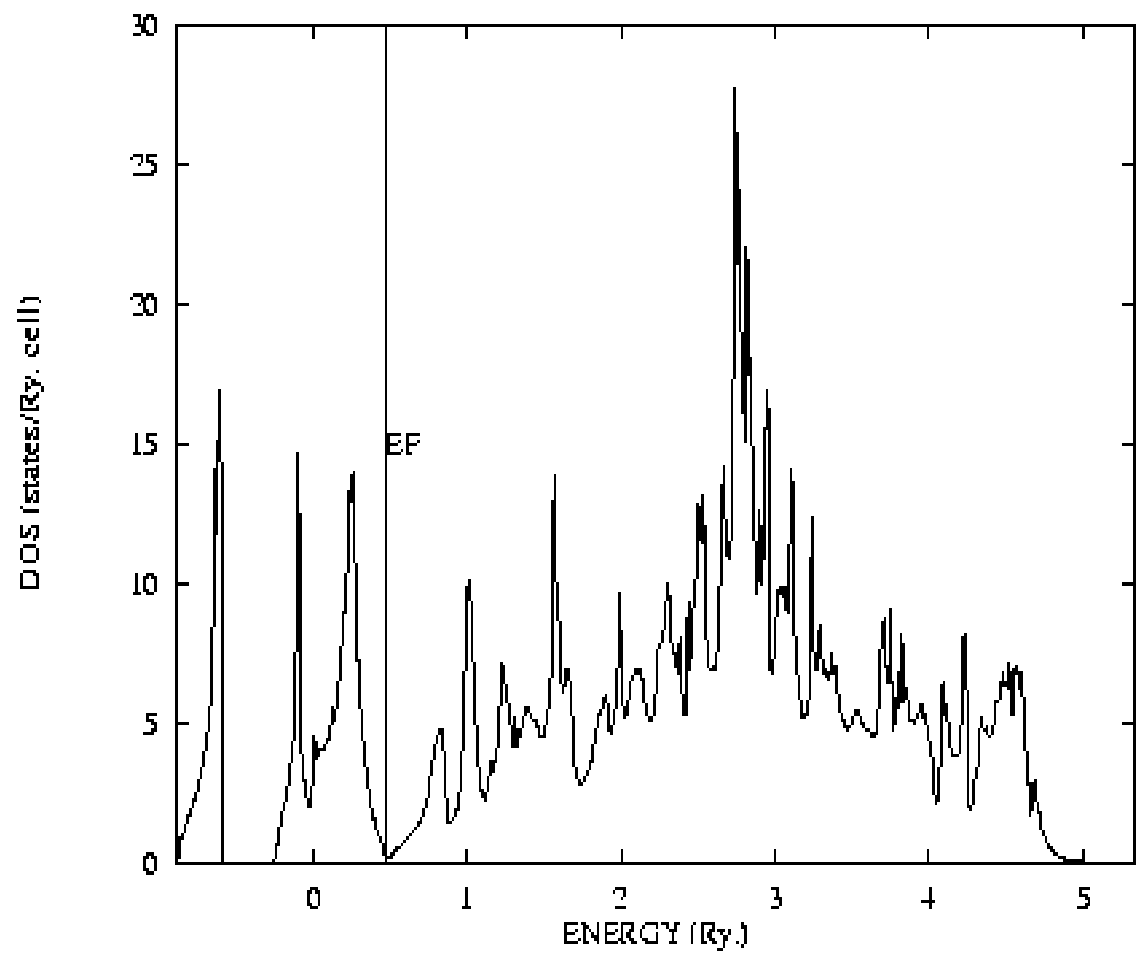

Figure 4. Density of states of $\mathrm{NaI}$ at $\mathrm{V} / \mathrm{Vo}=0.36(\mathrm{CsCl}$ structure $)$ 
When pressure increases (reduced volume decreases)the value of the $\mathrm{DOS}$ at fermi energy, $\mathrm{E}_{\mathrm{F}}\left(\mathrm{N}\left(\mathrm{E}_{\mathrm{F}}\right)\right.$ ) increases [17]. The normal pressure DOS trend is changed under high pressure. The increase of pressure leads to the broadening of bands and decrease of states value in most of the energy regions. It also increases the width of the valance band and empty conduction bands[14,15]. When pressure increases, the value of $E_{F}$ increases, whereas no density of states is available at the fermi level up to metallization pressure. Further increases in pressure leads to enhanced density of states at the fermi level which induces superconductivity[20].

\section{Result and Discussion}

\subsection{Ground State Properties}

From the total energies obtained from our calculation, the ground state properties and structural phase transition of $\mathrm{NaI}$ is studied. The total energy versus reduced volume curve is given in Fig.5. The total energy is calculated as a function of reduced volume $(\mathrm{V} / \mathrm{Vo})$ for both $\mathrm{NaCl}$ and $\mathrm{CsCl}$ phases of NaI. Here, Vo is the experimental equilibrium volume corresponding to the experimental equilibrium lattice constant. The calculated total energies were fitted to Murnaghan's equation of state [14]

$$
\begin{gathered}
\mathrm{P}=1.5 \mathrm{Bo}[(\mathrm{Vo} / \mathrm{V}) 7 / 3-(\mathrm{Vo} / \mathrm{V}) 5 / 3][1+0.75 \\
\left.\left(\mathrm{B}_{0}{ }^{1}-4\right)\{(\mathrm{Vo} / \mathrm{V}) 2 / 3-1\}\right]
\end{gathered}
$$

to obtain the pressure derivative of bulk modulus $\mathrm{Bo}^{1}$. The calculated reduced volumes, lattice constant and pressure values of $\mathrm{NaI}$ for different pressure are given in Table 1. The calculated total energies were fitted to Murnaghan's equation of state [8] to obtain the equilibrium lattice constant and other ground state properties. The values are given in Table 2. From the total energy as a function of reduced volume curve (Fig.5), it is found that, in NaI, up to $\mathrm{V} / \mathrm{Vo}=0.36\left(\mathrm{P}_{\mathrm{T}}=2.6259 \mathrm{Mbar}\right), \mathrm{NaCl}$ structure has the lowest energy and on further reduction of volume $\mathrm{CsCl}$ structure becomes more stable in energy than the $\mathrm{NaCl}$ structure (Table.3).

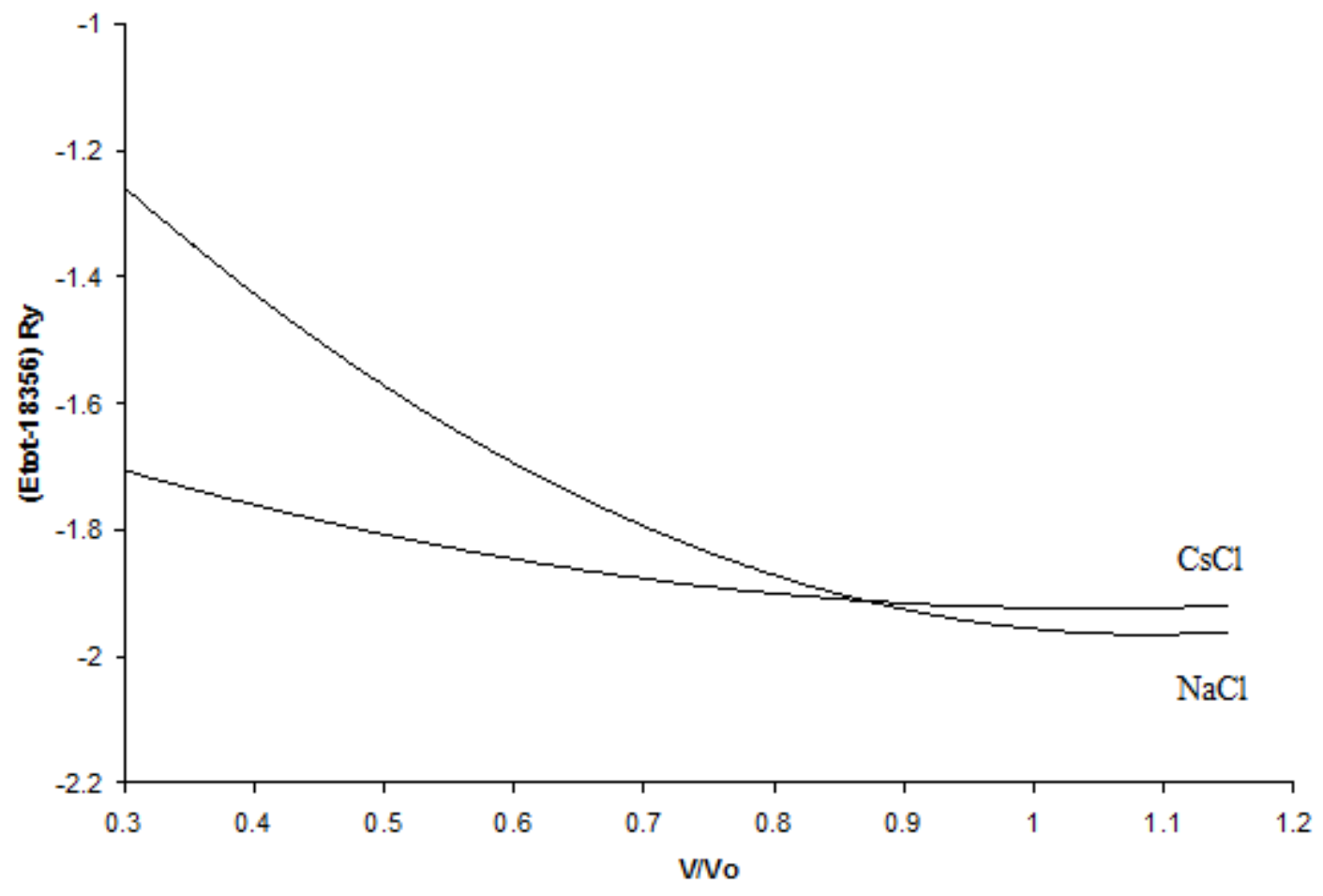

Figure 5. The relation connecting reduced volume and total energy of NaI 


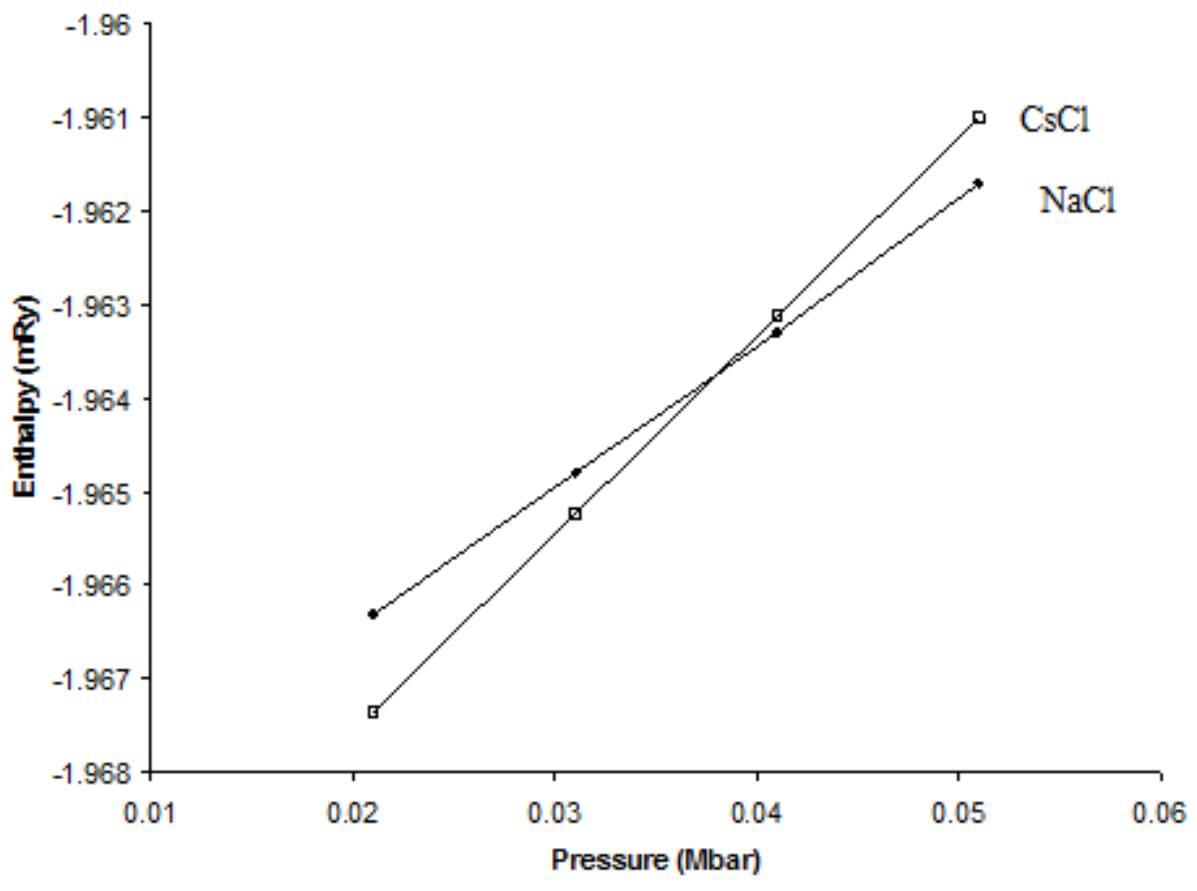

Figure 6. The relation connecting pressure and enthalpy of NaI

Table 1. Lattice constant (a) and Pressure (P) for different reduced volumes

\begin{tabular}{|c|c|c|c|}
\hline$V / V o$ & $a($ a.u $)$ & $P($ Mbar $)$ & $E_{g}(\mathrm{ev})$ \\
\hline 1 & 12.212 & 0 & 3.374 \\
\hline 0.9 & 11.791 & 0.0214 & 3.346 \\
\hline 0.8 & 11.366 & 0.0628 & 3.292 \\
\hline 0.7 & 10.843 & 0.0920 & 3.254 \\
\hline 0.6 & 10.299 & 0.3195 & 2.959 \\
\hline 0.5 & 9.6293 & 0.7154 & 2.444 \\
\hline 0.4 & 8.9979 & 1.7601 & 1.0859 \\
\hline 0.36 & 8.687 & 2.6259 & 0.0001 \\
\hline
\end{tabular}

Table 2. Equilibrium Lattice constant $\left(\mathrm{a}_{0}\right)$, Bulk modulus $\left(\mathrm{B}_{0}\right)$ and its Devrivative $\left(\mathrm{B}_{0}{ }^{1}\right)$

\begin{tabular}{|c|c|c|c|}
\hline Alkali iodide & $\mathrm{a}_{\mathrm{o}}$ (a.u) & $\mathrm{B}_{\mathrm{o}}$ (Mbar) & $\mathrm{B}_{\mathrm{o}}{ }^{1}$ \\
\hline $\mathrm{NaI}$ & 12.212 & 0.151 & 5.59 \\
\hline
\end{tabular}

Table 3. Metallization and Phase transition in Alkali iodides

\begin{tabular}{|c|c|c|c|}
\hline COMPOUND & Lattice constant $(\mathrm{au})$ & $(\mathrm{V} / \mathrm{V} 0)_{\mathrm{M}}$ & $\mathrm{P}_{\mathrm{M} M b a r}$ \\
\hline $\mathrm{NaI}$ & 12.212 & 0.36 & 2.626 \\
\hline $\mathrm{KI}[4]$ & 13.406 & 0.43 & 1.228 \\
\hline $\mathrm{RbI}[1]$ & 13.967 & 0.4 & 1.157 \\
\hline
\end{tabular}

\subsection{Structural Phase Transition}

At ambient pressure $\mathrm{NaI}$ is in the $\mathrm{NaCl}$ structure. The phase stability of the $\mathrm{B} 1(\mathrm{NaCl})$ and $\mathrm{B} 2(\mathrm{CsCl})$ structures of $\mathrm{NaI}$ is analyzed using the enthalpy calculation $[18,19]$. The enthalpy $\mathrm{H}(\mathrm{P})$ is defined by

$$
\mathrm{H}(\mathrm{P})=\text { Etot }(\mathrm{P})+\mathrm{PV}
$$

Where Etot $(\mathrm{P})$ is the total energy of $\mathrm{NaI}$ as a function of pressure $\mathrm{P}$ and $\mathrm{V}$ is the volume of $\mathrm{NaI}$ at pressure $\mathrm{P}$. The transition pressure corresponding to the phase transition from $\mathrm{B} 1$ to $\mathrm{B} 2$ is obtained from the relation

$$
\mathrm{H}_{\mathrm{B} 1}(\mathrm{P})=\mathrm{H}_{\mathrm{B} 2}(\mathrm{P})
$$

Where $\mathrm{H}_{\mathrm{B} 1}$ and $\mathrm{H}_{\mathrm{B} 2}$ aretheenthalpiesoftheB1 andB2phasesr espectively. The enthalpy versus pressure curve is given in Fig.6. In this figure, up to $\mathrm{P}=0.038 \mathrm{Mbar}, \mathrm{NaCl}$ has lowest energy. Further increase of pressure $\mathrm{CsCl}$ has lowest energy. The phase transition pressure $\left(P_{\mathrm{T}}\right)$ and the corresponding reduced volume $\left(V_{\mathrm{T}}\right)$ estimated in our calculation are $0.038 \mathrm{Mbar}$ and 0.89 respectively. Our calculated phase transition pressure is in good agreement with the experimental value of $0.035 \mathrm{Mbar}$. However, there is no experimental or theoretical study available for comparison at these pressures in NaI. But experimental and theoretical studies are available for comparison with alkali iodides $\mathrm{KI}$ and RbI [Table. 4]

Table 4. Comparison of Metallization and phase transition in alkali iodides

\begin{tabular}{|c|c|c|c|c|}
\hline \multirow{2}{*}{$\begin{array}{c}\text { Alkali } \\
\text { iodide }\end{array}$} & \multicolumn{2}{|c|}{ Metallization } & \multicolumn{2}{c|}{ Phase transition } \\
\cline { 2 - 5 } & $\mathrm{P}_{\mathrm{M}} \mathrm{Mbar}$ & $(\mathrm{V} / \mathrm{Vo})_{\mathrm{M}}$ & $\mathrm{P}_{\mathrm{T}} \mathrm{Mbar}$ & $(\mathrm{V} / \mathrm{Vo})_{\mathrm{T}}$ \\
\hline $\mathrm{NaI}$ & 2.626 & 0.36 & 0.038 & 0.89 \\
\hline $\mathrm{KI}[4]$ & 1.228 & 0.43 & 0.025 & 0.92 \\
\hline $\mathrm{RbI}[1]$ & 1.157 & 0.4 & 0.0223 & 0.934 \\
\hline
\end{tabular}

\subsection{Metallization}

At normal pressure, $\mathrm{NaI}$ is a wide gap insulator (Fig. 1 and 2). As pressure is increased, there is a charge transfer from $s, p$ to $d$ state, this causes the increase in the width of 
the valence band and also the empty conduction bands. These changes lead to the narrowing of the band gap and at particular pressure, there is a closing of band gap. NaI becomes metal under pressure but before that it undergoes structural phase transition from B1 phase to $\mathrm{B} 2$ phase. The band structure and density of state corresponding to metallization of $\mathrm{NaI}$ is shown in Fig. 3 and 4 . In NaI, metallization takes place by the indirect closure (Fig. 3) of band gap between valence band maximum at $\Gamma$ point and conduction band minimum at the $\mathrm{H}$ point. The metallization volume of $\mathrm{NaI}$ is $V / V o=0.36$ which corresponds to the pressure $P_{M}=2.6259 \mathrm{Mbar}$. The metallization occurs because of the closure of band gap between I-5p- like valence band and Na-3s- like conduction band (Fig. 3). The increase of pressure causes the broadening of bands which results in the decrease of density of states value in most of the energy regions of DOS histograms. Thus in Fig. 4, the heights of the spikes is considerably reduced. When pressure is increased $E_{F}$ increases whereas no density of states is available at the Fermi level up to metallization pressure [5]. There are appreciable values for $\mathrm{DOS}$ at $\mathrm{V} / \mathrm{Vo}=0.36$ (Fig. 4) indicating metallization in NaI. The comparison of Metallization and phase transition in alkali iodides are given in Table.4. From tables 3 and 4, it is noted that, when lattice constant decreases metallization pressure and phase transition pressure increases.

\subsection{Superconductivity and Its Pressure Variation}

The continuous promotion of $s$, $p$ electron to $d$ shell in solids under pressure is one of the factors which will induce superconductivity [11]. The theory of Gaspari and Gyoffy in conjunction with McMillan's formula is used to calculate $T c$.

The electron -phonon mass enhancement factor, $\lambda$ is

$$
\lambda=\mathrm{N}\left(\mathrm{E}_{\mathrm{F}}\right)\left\langle\mathrm{I}^{2}\right\rangle / \mathrm{M}\left\langle\omega^{2}\right\rangle
$$

where $M$ is te atomic mass, $\left\langle\omega^{2}\right\rangle$ is an average of the phonon frequency square and $\left\langle\mathrm{I}^{2}\right\rangle$ is the square of the electron-phonon matrix element averaged over the Fermi surface. $\left\langle\mathrm{I}^{2}\right\rangle$ (in Rydbergs) can be written as [11].

$$
\left.\left\langle\mathrm{I}^{2}\right\rangle=2 \Sigma_{1}\{(\mathrm{l}+1) /(2 \mathrm{l}+1)(2 \mathrm{l}+3))\right\} \mathrm{M}_{1,1+1}^{2}\left\{\begin{array}{r}
\mathrm{N}_{1}\left(\mathrm{E}_{\mathrm{F}}\right) \mathrm{N}_{1+1}\left(\mathrm{E}_{\mathrm{F}}\right) / \\
\left.\mathrm{N}\left(\mathrm{E}_{\mathrm{F}}\right)^{2}\right\}(5)
\end{array}\right.
$$

Where,

$$
\mathrm{M}_{1,1+1}=\Phi_{1} \Phi_{1+1}\left[\left(\mathrm{D}\left(\mathrm{E}_{\mathrm{F}}\right)-1\right)\left(\mathrm{D}_{1+1}\left(\mathrm{E}_{\mathrm{F}}\right)+1+2\right)+\left(\mathrm{E}_{\mathrm{F}}-\mathrm{V}(\mathrm{S}) \mathrm{S}^{2}\right](6)\right.
$$

$\Phi_{1}$ is the radial wave function at the Muffin-Tin sphere radius corresponding to the Fermi energy.

$\mathrm{D}_{1}$ is the logarithmic derivative of the radial wave function at the sphere boundary.

$\mathrm{V}(\mathrm{S})$ is the Muffin - Tin potential at the sphere boundary. $\mathrm{S}$ is the radius of the Muffin - Tin sphere.

The above quantities to calculate $\mathrm{M}_{1,1+1}$ are taken from band structure results[11]. We have calculated $\chi$ separately for $\mathrm{Na}$ and $\mathrm{I}$ atoms and for the $\mathrm{T}_{\mathrm{c}}$ calculation (Eq.(7)) the mean value of is used

The average of the phonon frequency square is

$$
\left\langle\omega^{2}\right\rangle=0.5 \theta_{\mathrm{D}}^{2}
$$

The variation of Debye temperature with pressure $\theta_{D}(P)$ is given by [11]

$$
\theta_{\mathrm{D}}(\mathrm{P})=\theta_{\mathrm{D}}^{0}\left(\mathrm{a}_{0} / \mathrm{a}\right)\left(\sqrt{\mathrm{E}_{\mathrm{F}}} / \mathrm{VE}_{\mathrm{F}}^{0}\right)
$$

where $\theta_{D}{ }^{o}, a_{o}$ and $E_{F}{ }^{o}$ are normal pressure quantities. McMillan's formula,

$$
\mathrm{Tc}=\left(\theta_{\mathrm{D}} / 1.45\right) \exp \left\{-1.04(1+\lambda) /\left[\lambda-\mu^{*}(1+0.62 \lambda)\right]\right\}
$$

gives the good estimate of the $T c$ value [31]. Here $\mu^{*}$ is the electron-electron interaction parameter which is estimated using the relation,

$$
\mu^{*}=0.26 \mathrm{~N}\left(\mathrm{E}_{\mathrm{F}}\right) /\left(1+\mathrm{N}\left(\mathrm{E}_{\mathrm{F}}\right)\right)
$$

where

$N\left(E_{F}\right)$ is the density of levels per atom per eV at $E_{F}$.

The Fermi energy $E_{F}(\mathrm{Ry})$ and density of states $N\left(E_{F}\right)$ (states/Ry.cell) are obtained from the self-consistent calculation and we have calculated the variation of $\theta_{D}, \lambda$ and $T c$ with pressure using Eqs.(5-8) [21]. The value of $T c$ is determined at higher pressures also. The calculated values for $\theta_{D}, \lambda$ and $T c$ under various pressures are given in Table.5.In our calculation the highest $T c$ obtained is 1.18708 at 0.3 Mbar. The calculated Tc values depends more sensitive lyon $\lambda$ rather than $\theta_{D}$ [21]. For NaI, no experimental Tc value available for comparison.

Table 5. $T c$ as a function of pressure for $\mathrm{NaI}$

\begin{tabular}{|c|c|c|c|c|}
\hline Pressure $P$ Mbar & $\lambda$ & $\theta_{D} \mathrm{~K}$ & $\mu^{*}$ & $T c \mathrm{~K}$ \\
\hline 3.6241 & 0.164 & 275 & 0.026 & 0.02467 \\
\hline 4.0551 & 0.197 & 297 & 0.04 & 0.05717 \\
\hline 4.5501 & 0.249 & 309 & 0.051 & 0.22688 \\
\hline 45.1211 & 0.363 & 321 & 0.075 & 1.18708 \\
\hline
\end{tabular}

\section{Conclusions}

The high pressure band structure, density of states, structural phase transition, metallization and superconductivity of $\mathrm{NaI}$ is investigated. When the pressure is increased there is enhanced overlapping between the wave functions of the neighbouring atoms. As a result the widths of the valence and empty conduction bands increase. These changes lead to the narrowing and closing of band gaps (metallization). The metallization reduced volume is $V / V_{o}=0.36(\mathrm{CsCl}$ structure), and the corresponding pressure $P_{M}$ is 2.6259 Mbar. In our calculation $\mathrm{NaCl}$ phase to $\mathrm{CsCl}$ phase transition occurs at 0.038 Mbar. This value is good agreement with the experimental value of $0.035 \mathrm{Mbar}$. It is also confirmed that the structural phase transition and metallization do not occur simultaneously in alkali iodide compounds [3].

\section{Acknowledgement}

The authors are thankful to the University grant 
commission (UGC) for the financial assistance through the Minor Research Project No: 6815/16(SERO/ UGC).

\section{REFERENCES}

[1] C. Nirmala Louis and Y. Ramola, International Journal of Chemical Science, 2(1), (2018) 36-41.

[2] A. Amalraj, C. Nirmala Louis and Sr. Gerardin Jayam, Journal of Theoretical and Computational Chemistry, Vol. 6, No. 4 (2007) 833-843.

[3] M. I. Eremets, K. Shimizuand K. Amaya Science 281(1998)1333.

[4] C. Nirmala Louis and K. Iyakutti, Physica status solidi (b), 233, 339-350 (2002).

[5] S. Q. Wang and H. Q. Ye, J. Phy. Condensed matter 14 (2002) 9579.

[6] C. Nirmala Louis, K. Iyakutti, K. S. Hema Nandhini, M. Sivakumar and E. Palaniyandi, Physica Status Solidi (b) 241, (2004)2489-2500;

[7] U. von Barth and L. Hedin, J. Phys. C 5 (1972) 629.

[8] F. D. Murnaghan, Proc. Natl. Acad. Sci. USA 30 (1944) 244.

[9] G.D. Gaspariand B. L. Gyorffy, Phys .Rev. Lett29 (1972) 801.

[10] W. L. Mcmillan, Phys. Rev. 167(1968) 331.

[11] Y. Ramola, C. Nirmala Louis and A. Amalraj, Chemical and Materials Engineering (2017), 5(3): 65-74.

[12] Y. K. Vohra, S. T. Weir, K.E. Brister and A. L. Ruoff, phys. Rev. Lett 55, 977(1985).

[13] S. T. Weir, J. Akella and C. Aracne-Ruddle, Y. K. Vohraand S. A. Catledge, Appl. Phys. Lett. 77, 3400 (2000).

[14] J. Jesse Pius, A. Lekshmiand C. Nirmala Louis, Chemical and Materials Engineering 5(1): 8-13, 2017.

[15] V. Benaline Sheeba and C. Nirmala Louis, International Journal of Scientific Research and Innovations, 1, 1-5(2016).

[16] O. K. Andersen, O. Jepsen and M. Sob, "Electronic band structure and its applications", edited by M. Yussouff (Springer, Heidelberg,1987)

[17] R. Asokamani, G. Pari, R. Rita, and R. Mercy, phys. Stat. Sol.(b) 199, 157 (1997). R. Asokamani and C. Ravi, Bull. Mater. Sci. 22, 101 (1999).

[18] M. Rajagopalan, C. U. M. Trinath, and S. Natarajan, J. Alloys Compd. 274, 18(1998).

[19] Russel .J. Hemley, Science 281, 1296(1998).

[20] V. Benaline Sheeba and C. Nirmala Louis, Chemical and Materials Engineering 1(4): 132-140, 2013. 